\title{
ANÁLISIS DEL COMPORTAMIENTO DE CONDUCTORES EN CURVAS DE BAJO ÁNGULO DE DEFLEXIÓN
}

\author{
José Luis Ulpiano Cogollos \\ Grupo de Investigación en Ingeniería de Carreteras (GIIC), Universitat Politècnica de \\ València, España \\ Francisco Javier Camacho-Torregrosa \\ Profesor Ayudante Doctor, GIIC, Universitat Politècnica de València, España
}

Alfredo García García

Catedrático, GIIC, Universitat Politècnica de València, España

\begin{abstract}
RESUMEN
Las curvas con un bajo ángulo de deflexión pueden ser un problema para la percepción por parte de los conductores. Para evitar este aspecto, las diferentes normativas establecen criterios de desarrollo o radios mínimos, que las haga apreciables por los conductores. Los criterios que están incorporados en las normativas españolas nunca tuvieron una base experimental científica sino fueron fruto de juicios técnicos.
\end{abstract}

Los objetivos de esta investigación son analizar la percepción subjetiva y caracterizar la respuesta de los usuarios de carreteras convencionales ante curvas de bajo ángulo de deflexión, para conseguir unas recomendaciones de diseño para este tipo de curvas. Estas curvas se restituyen geométricamente con el objetivo de identificar cuál servirá para un posterior estudio más detallado in situ.

Para ello se presenta el diseño y desarrollo de un cuestionario online, el cual incorpora una base de datos de curvas de bajo ángulo de deflexión presentes en carreteras convencionales españolas.

El cuestionario consiste en mostrar a un amplio número de conductores una serie de vídeos aleatorios donde se visualiza, desde el punto de vista del usuario de la carretera, el recorrido de varias de las curvas. Tras este paso, los encuestados deben expresar su percepción y comportamiento a través de varias preguntas de tipo abierto y cerrado. Estos resultados quedan registrados en una base de datos, para su posterior filtrado, tratamiento e interpretación estadística. 


\section{INTRODUCCIÓN}

El diseño geométrico de carreteras que actualmente se aplica en España se basa fundamentalmente en la Instrucción de Trazado (Ministerio de Fomento, 1999). Dicha Instrucción ofrece unos criterios básicos para crear un trazado con condiciones adecuadas de seguridad y comodidad, pero sin una base experimental científica.

El diseño de curvas de bajo ángulo de deflexión debería realizarse considerando diferentes criterios, entre los que deberían incluirse aspectos funcionales, operacionales, de seguridad vial y de percepción por parte de los conductores. Si bien todos ellos están altamente ligados, el enfoque necesario difiere en metodología y tipo de datos a considerar, siendo los resultados complementarios.

En cuanto a los aspectos operacionales, podemos distinguir entre trayectorias y velocidades de operación. Respecto de las trayectorias recorridas por los vehículos, una de las investigaciones más relevantes sobre trayectorias vehiculares en curvas fue la conducida por (Spacek, 2005), quien clasificó las trayectorias más comunes de los conductores en seis tipos, identificando tendencias en función de su configuración geométrica y la existencia o no de clotoides. La maniobra más común fue la de recortar la curva. Sin embargo, esta investigación no estaba centrada en la particularidad de las curvas de bajo ángulo, que bien merecen un estudio particular. Otra investigación similar fue la conducida por (Fitzsimmons et al. 2013), obteniendo resultados similares.

En cuanto a la velocidad de operación, la gran mayoría de los modelos utiliza el radio para estimar a qué velocidad circulan los conductores en las curvas. Esto se traduce en que curvas con un radio muy elevado sí pueden estar bien recogidas por dichos modelos, pero no así curvas de radios menores pero con un desarrollo muy reducido. En estos casos, sería necesario recurrir a modelos dependientes de otros parámetros, como el radio y la longitud como el modelo de (Ottesen, 2000 ; Krammes, 2000) o del CCR (Curvature Change Ratio), como el propuesto por (Pérez-Zuriaga. 2012).

En cuanto a la funcionalidad, si bien la actual Instrucción de Trazado 3.1 IC (2016) no incluye criterios de funcionalidad propios en su diseño, sí hace referencia a documentos externos, como el Highway Capacity Manual (AASHTO, 2010). En este documento no hay referencia explícita al tratamiento de las curvas de bajo ángulo de deflexión y su impacto sobre la funcionalidad.

En la actualidad, no se ha referido de forma explícita un aumento de la siniestralidad en este tipo de curvas, si bien su apariencia puede dar lugar en ocasiones a una falsa percepción por parte de los conductores, produciendo maniobras erráticas.

Para curvas circulares de radio menor que cinco mil metros en carreteras del grupo uno y dos mil quinientos metros en carreteras del grupo dos, será necesario utilizar curvas de 
transición, mientras que para curvas circulares de radios mayores o iguales que los indicados no será necesario utilizarlas.

En el caso de valores excepcionales de ángulos de giro entre rectas inferiores a seis gonios y desarrollo de la curva para mejorar la percepción visual, se realizará la unión de las mismas mediante una curva circular, sin clotoides, de radio tal que se cumpla (1)

$$
\text { Desarrollo de la curva } \geq 325-25 \cdot \Omega
$$

Para ángulos de giro entre rectas ligeramente superiores a seis gonios, se comprobará siempre que la suma de las longitudes de las curvas de transición y de la curva circular, sea superior a unos desarrollos mínimos

\section{OBJETIVOS}

Con el objetivo de conocer con mayor profundidad la afección que estas curvas de bajo ángulo de deflexión tienen sobre la funcionalidad y seguridad de carreteras convencionales, el Grupo de Investigación en Ingeniería de Carreteras (GIIC) de la Universitat Politècnica de València está llevando a cabo una investigación al respecto. Dicha investigación es una de las partes en las que está dividido el proyecto "CASEFU - Estudio experimental de la funcionalidad y seguridad de las carreteras convencionales".

En dicha investigación se abordará el estudio de las curvas de bajo ángulo de deflexión desde dos puntos de vista: analizando en campo las trayectorias de los vehículos en estas curvas, así como realizar una encuesta on-line para recoger la percepción de los conductores frente a diferentes curvas de bajo ángulo.

En el presente documento se recoge el desarrollo de la segunda de estas investigaciones, es decir, el planteamiento y desarrollo de una web que sirva para encuestar a un gran número de personas sobre la percepción de diferentes curvas de bajo ángulo de deflexión. Los datos recogidos serán almacenados en una base de datos online, para después ser descargados, filtrados e interpretados.

\section{METODOLOGÍA}

La primera fase de esta investigación puede dividirse en las siguientes dos partes claramente diferenciadas:

- Obtención de una muestra suficiente de curvas de bajo ángulo de deflexión a lo largo de la geografía española.

- Creación y alojamiento del cuestionario web

\subsection{Obtención de la muestra de curvas a analizar}

Esta fase comienza con la detección de un gran número de curvas de bajo ángulo de deflexión dentro del territorio nacional. Mediante la revisión de ortofotografías aéreas, se ha creado 
una base de datos de 576 curvas que potencialmente pueden ser de bajo ángulo de deflexión esto es, entre dos y veinte gonios

Para cada una de esas curvas, el primer paso consiste en restituirlas geométricamente, determinando así si puede considerarse de bajo ángulo de deflexión y, en dicho caso, sus propiedades geométricas. Es importante señalar la dificultad de restituir geométricamente curvas tan suaves, ya que el rango de radios y parámetros que dan lugar a soluciones aproximadamente válidas es muy grande. Con el objetivo de limitar dicha variabilidad, se ha procedido a aplicar un procedimiento automático de restitución geométrica basado en el análisis del azimut, presentado por (Camacho-Torregrosa et al. 2015). El empleo de esta metodología consigue un grado de ajuste muy superior a los que se pueden conseguir mediante otros métodos.

También es importante señalar que no interesan únicamente curvas de bajo ángulo de deflexión, sino también curvas que no son consideradas como tal pero que están cerca de serlo.

Además de las propiedades geométricas, también hay que tener en cuenta otros criterios para seleccionar o no cada una de las curvas. Por ejemplo, la presencia de accesos en la curva o sus inmediaciones es criterio de exclusión de la curva, al igual que una sección transversal de características claramente insuficientes.

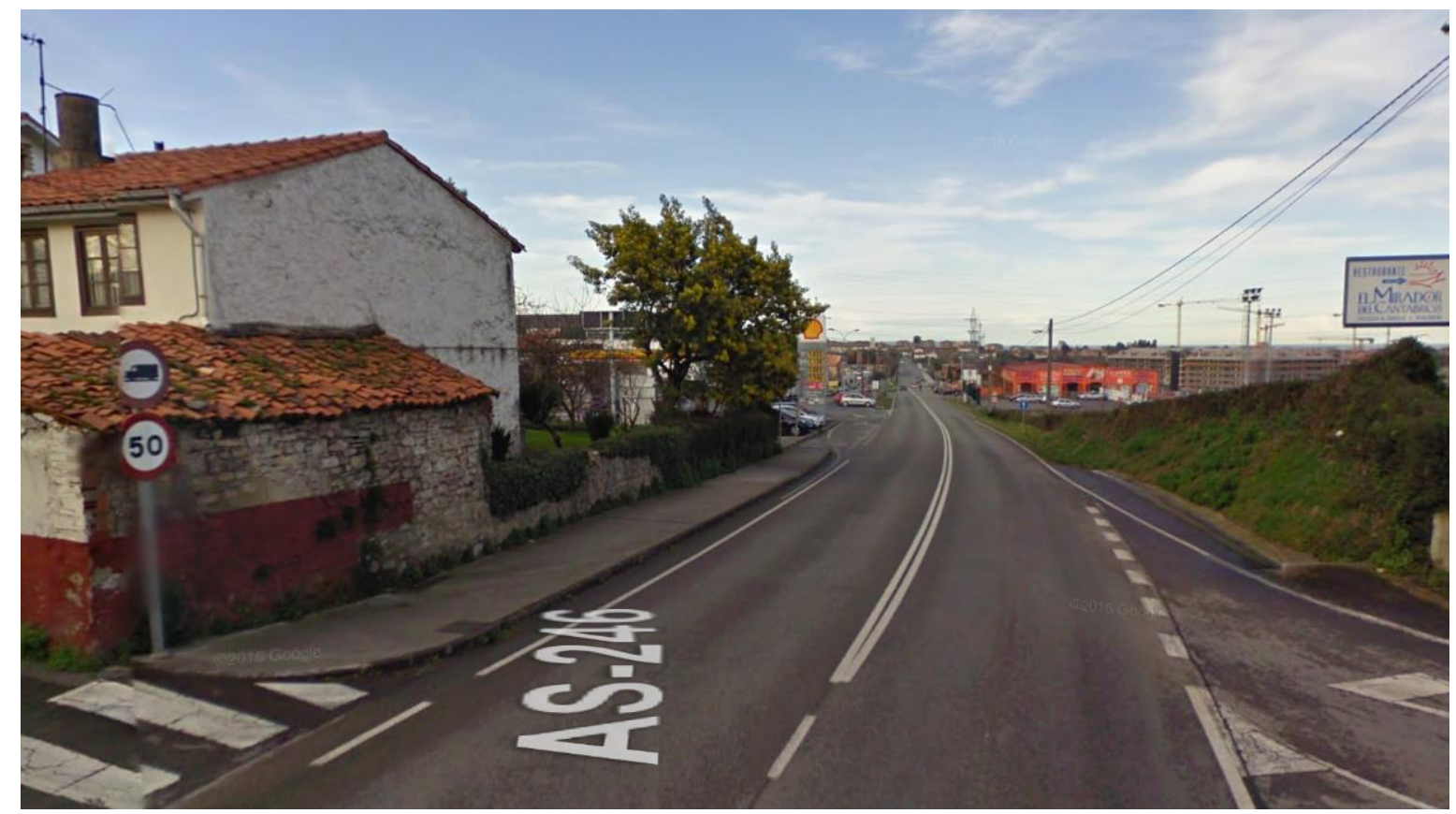

Figura 1 - Curva de bajo ángulo de deflexión, periurbana y con intersección. 


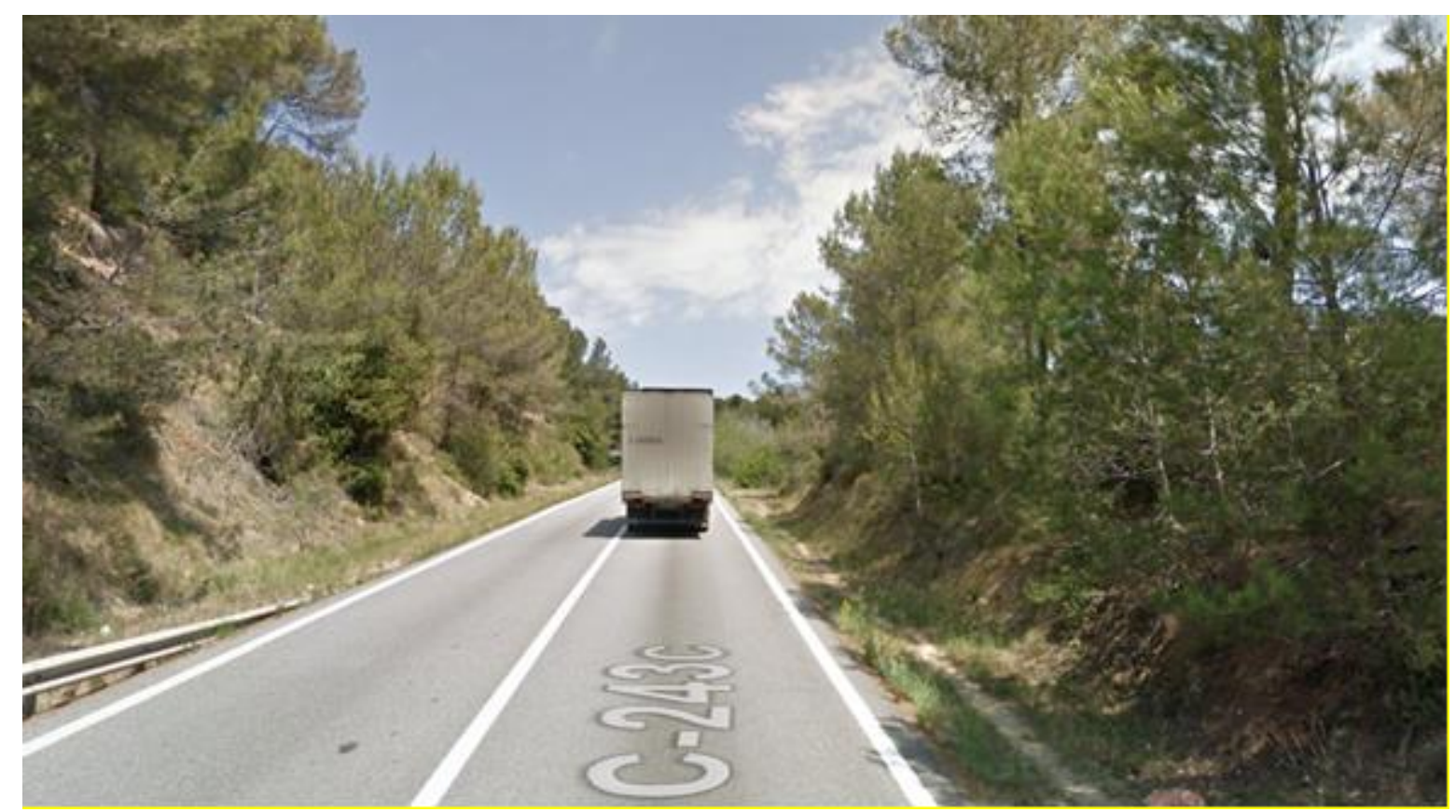

Figura 2 - Curva de bajo ángulo de deflexión, con camión delante.

\subsection{Creación del cuestionario online}

El cuestionario online debe mostrar una serie de vídeos del recorrido de curvas de bajo ángulo de deflexión. Tras cada vídeo deben seguir unas preguntas acerca de la opinión de los usuarios sobre el vídeo que acaban de visualizar.

El cuestionario debe presentar las siguientes características:

- Puesto que el número de curvas es muy elevado, con propiedades variadas, los vídeos que se muestran deben ser escogidos de forma aleatoria de la base de datos con todos ellos.

- El cuestionario debe poder ser abierto por los exploradores más extendidos a día de hoy (Internet Explorer, Edge, Google Chrome y Mozilla Firefox).

- El cuestionario debe de poder ser cargado rápidamente, y no requerir ningún tipo de instalación de software adicional. Esto incluye el envío automático de los resultados, sin intervención por parte del encuestado.

- El tiempo estimado de realización del cuestionario debe ser analizado meticulosamente. Cuestionarios de muy corta duración permitirán recoger muy pocos datos, mientras que cuestionarios muy largos implicarán una mayor tasa de abandono.

- Debe recoger otros datos sociológicos, que no permitan identificar al encuestado (debe ser anónimo), pero sí encuadrarlo en grupos, como profesión, edad aproximada, experiencia al volante, etc.

Para cada una de las curvas a incluir en el estudio hay que realizar un vídeo que simule la visión que tendría un conductor que fuera circulando por ella. La restricción más importante 
de estos es que estén situados sobre la carretera, que se aprecie esta y su entorno de forma nítida, y que no exista tráfico u otros obstáculos que impidan al encuestado su correcta visualización.

\section{DESARROLLO}

\subsection{Análisis de la muestra de curvas disponible}

Actualmente se dispone de la geometría de 72 curvas, lo que puede dar una primera idea sobre la potencial problemática de este tipo de curvas en nuestra red de carreteras.

La Figura 3 muestra la distribución de desarrollos completos de las curvas en función del ángulo girado. Podemos distinguir cuatro zonas:

- Zona sin sombreado: curvas normales, con un desarrollo superior a veinte gonios.

- Zona con sombreado verde: la Instrucción recomienda utilizar curvas de más de veinte gonios, pero permite aquellas que tienen entre seis y veinte. En este caso, las curvas deben diseñarse de forma convencional.

- Zona con sombreado amarillo: para curvas entre dos y seis gonios, la Instrucción permite colocar únicamente curvas circulares (es decir, sin clotoides), con un cierto desarrollo mínimo.

- Zona con sombreado rojo: zona de curvas que incumplen el desarrollo mínimo para curvas de deflexión entre dos y seis gonios, así como curvas de menos de dos gonios, no permitidas por la actual Instrucción.

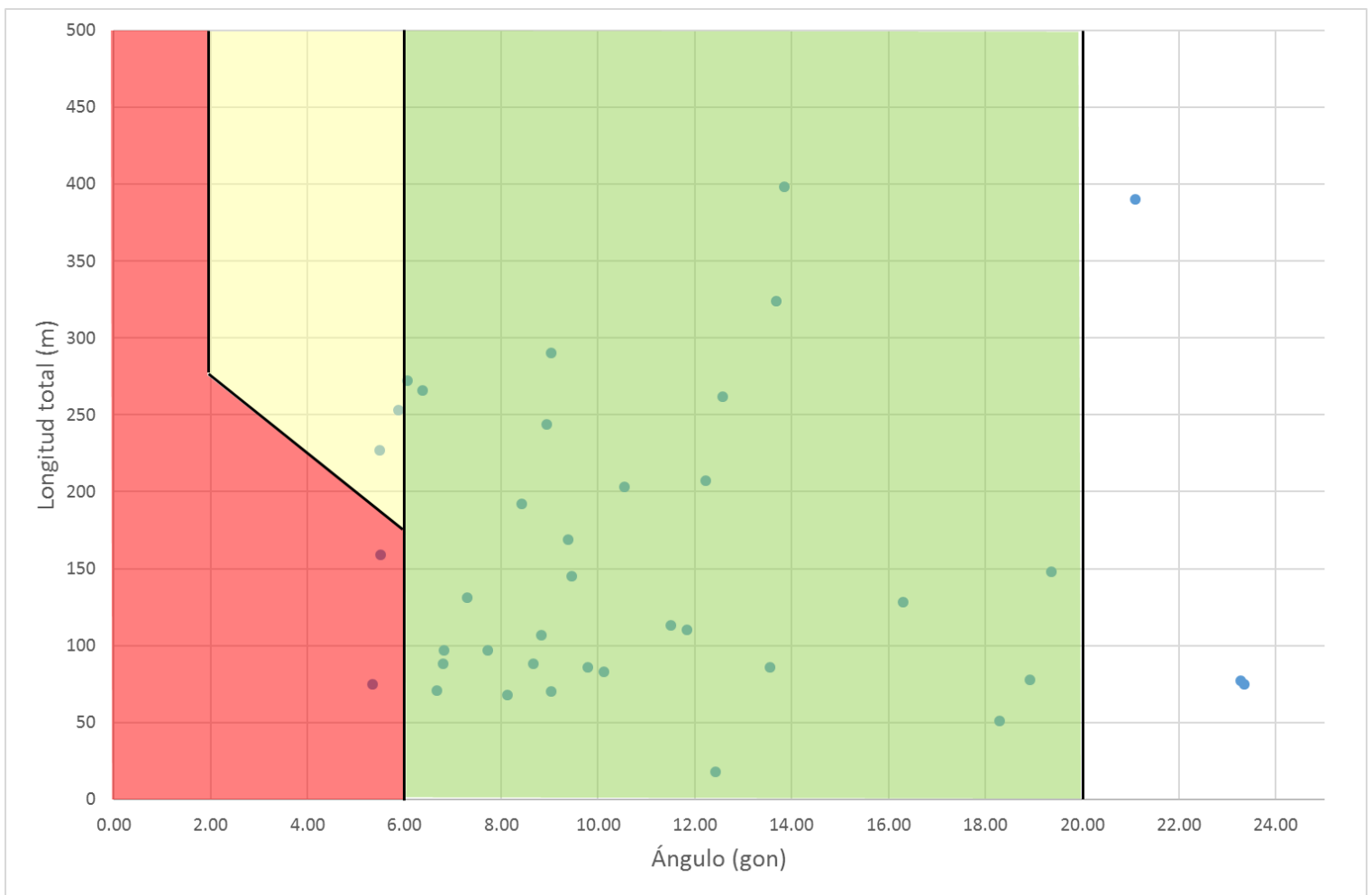

Figura 3 - Distribución de ángulos de deflexión y desarrollos. 
Como se puede ver en la Figura 3, la gran parte de curvas analizadas se encuentran en el rango seis-veinte gonios. Unas pocas están por debajo de seis gonios, de las cuales dos incumplen la actual Instrucción por tener un desarrollo inferior al mínimo. En la carretera, estos casos podrían aparentar un quiebro en el trazado.

\subsection{Desarrollo del cuestionario online}

Se valoraron inicialmente diferentes opciones para el cuestionario online. En un primer momento se buscó y se analizó distintas plataformas para confeccionar este cuestionario online. Puesto que ninguna de ellas permitía alojar vídeos y disponerlos de forma aleatoria, se concluyó que se precisaba programarla de cero.

Para programar la plataforma web, se analizaron diferentes opciones, llegando finalmente a utilizar el lenguaje ASP.NET para la web y SQL para las bases de datos.

Se cuentan con dos bases de datos. La primera de ellas, cerrada al usuario, tiene los siguientes campos:

- Identificación de la curva.

- Ruta al vídeo de Youtube correspondiente.

- Identificador que sirve para indicar si la curva a izquierda o derecha.

Este cuestionario no caracteriza al usuario participante, siendo totalmente anónimo, confidencial y no agregado (no permite identificar a nadie, pero sí existe un registro único para cada respuesta).

Todos los datos facilitados a través del cuestionario electrónico son tratados con estricta confidencialidad de acuerdo con la Ley de Protección de Datos de Carácter Personal.

Para cada vídeo se han planteado las siguientes preguntas, junto con las correspondientes opciones cerradas de respuesta:

- ¿Puede ver correctamente el trazado de la curva?

- Sí, el trazado se visualiza correctamente

- No, hay elementos externos que impiden ver correctamente el trazado

- Otros (especificar)

- ¿Le parece que el diseño geométrico es adecuado?

- Sí, el diseño es adecuado

- No, en la curva se aprecia un quiebro o discontinuidad del trazado

- No, a medida que me acerco parece que cambia su sentido de giro

- Otros (especificar)

- ¿Cómo cree que recorrería la curva en términos de trayectoria? (siempre que el tráfico y las condiciones externas lo permitan) 
- Seguiría centrado en mi carril, como usualmente conduzco

- Probablemente tendería a recortar ligeramente la curva, sin salir de mi carril

- Probablemente tendería a recortar ligeramente la curva invadiendo el carril contrario/arcén

○ Otros (especificar)

- ¿Cómo cree que recorrería la curva en términos de velocidad? (siempre que el tráfico y las condiciones externas lo permitan)

- Mantendría la misma velocidad que en la recta, pues no ofrece problemas para ello

- Reduciría ligeramente la velocidad

- Reduciría la velocidad por una causa diferente, no asociada a la curva en sí (especificar el motivo)

- Otros (especificar)

Como se ve, en todos los casos aparece una opción para que el usuario introduzca comentarios en caso de que lo considere necesario.

Considerando estas preguntas, se decidió incluir un total de cinco vídeos con sus correspondientes preguntas. Cada vídeo puede reproducirse las veces que el usuario considere oportunas.

Previamente, a los usuarios se les pregunta por los siguientes datos sociológicos:

- País

- Sexo

- Nivel de estudios

- Área de conocimiento

- Edad

- Experiencia al volante

- ¿Cuántos kilómetros recorre al año como conductor, aproximadamente?

- ¿Qué tipo de vehículo conduce habitualmente?

Al finalizar el cuestionario, también se pregunta acerca de otros datos más relacionados con su actividad como conductor:

- ¿Conoce cuántos puntos tiene en su carnet?

- ¿Ha recibido alguna multa que no sea de aparcamiento?

- ¿Ha estado implicado en algún accidente de circulación?

Las primeras preguntas buscan agrupar las respuestas en función del tipo de usuario. Las últimas, persiguen poder identificar conductas más agresivas en este tipo de curvas con el historial de infracciones o accidentes. 
En cuanto a cada uno de los vídeos, la mejor opción sería grabar cada secuencia utilizando una cámara a la altura del conductor mientras se circula por cada una de las curvas. Este planteamiento es inviable económicamente, puesto que son más de quinientas las curvas incluidas en el análisis, repartidas por todo el país. Por ello se decidió hacer uso del servicio Google Street View, creando pequeños vídeos que muestran la secuencia de fotogramas de cada recorrido a intervalos de tiempo regulares.

Cada vídeo comienza en la recta anterior de la curva, a una distancia suficiente como para que el encuestado pueda observar el entorno y adaptarse a él. Quedan, por tanto, excluidas las curvas de bajo ángulo con rectas previas de muy pequeño desarrollo. Los vídeos finalizan poco después de que lo haga la curva de bajo ángulo. La nitidez es importante controlarla en este proceso, así como la orientación de cada uno de los fotogramas, con el objetivo de evitar el desconcierto de los encuestados. La duración aproximada de los vídeos se encuentra entre diez y veinte segundos. Cada uno de ellos se crea de forma automática y se aloja en Youtube. De esta forma se consigue una carga muy rápida de la web (al no tener que subir vídeos a la plataforma), así como el menor consumo de recursos propios de espacio digital. La contrapartida estriba en que es necesario tener instalado el complemento Flash Player, si bien a día de hoy su uso está completamente extendido. Las Figuras 4 y 5 muestran dos capturas de pantalla del cuestionario en su estado actual.

\section{CUESTIONARIO}

UNIIIVRSIDAD
POLITECNICA
DE VALENCIA

\section{Objetivo de la investigación}

El diseño de carreteras convencionales en muchas ocasiones precisa unir dos rectas por medio de una curva con muy poco ángulo de giro, conocida como "curva de bajo ángulo de deflexión".

Este tipo de curvas suele presentar la problemática de que los conductores pueden percibirla, cuando se aproximan a ella, como un quiebro o discontinuidad en el trazado.

Este problema puede resolverse aumentando su radio.

La actual Norma de Trazado en España incorpora un criterio para controlar dicho radio, si bien no existen investigaciones sobre su efectividad en términos operacionales o de seguridad.

El objetivo de la presente investigación es recopilar información sobre el efecto que las curvas de bajo ángulo de giro producen sobre los conductores.

En esta fase del estudio se pretende obtener la percepción de diversos conducotres al visualizar curvas de diferentes características. El objetivo último será actualizar las recomendaciones para lograr un mejor diseño.

\section{Figura 4 - Instrucciones iniciales del cuestionario.}


¿Puede ver correctamente el trazado de la curva?

-Seleccione una opción-

¿Le parece que el diseño geométrico es adecuado?

-Seleccione una opción-

¿Cómo cree que recorrería la curva en términos de trayectoria? (siempre que el tráfico y las condiciones externas lo permitan)

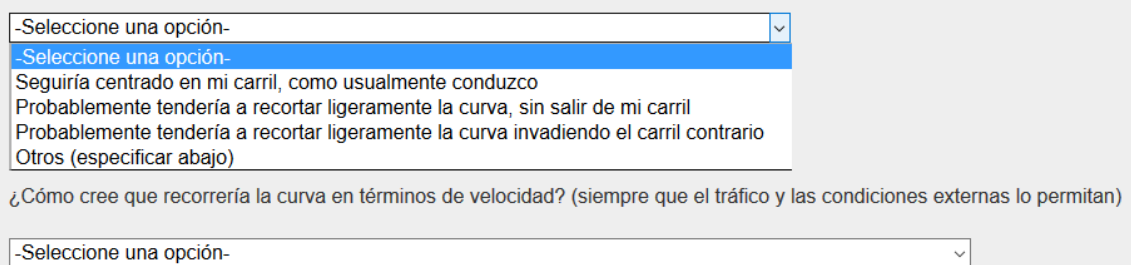

-Seleccione una opción-

Figura 5 - Captura del cuestionario con las opciones de una pregunta desplegadas.

\section{CONCLUSIONES}

Se ha presentado el estado actual de la investigación que se está realizando con el fin de analizar la percepción de los conductores a diferentes curvas de bajo ángulo de deflexión. La metodología de toma de datos consiste en una encuesta web a un gran número de personas de diferentes características, que responderán a preguntas acerca de la percepción visual de diferentes curvas, así como a cómo conducirían en dichas condiciones. El objetivo final es, junto con otros criterios de seguridad, operación y funcionalidad, dar unas recomendaciones fundamentadas para establecer unos criterios de diseño de curvas de bajo ángulo de deflexión, más allá de los existentes hoy día en la Instrucción de Trazado 3.1 IC.

\section{FUTURA INVESTIGACIÓN}

Actualmente el cuestionario está editado y subido a la web. Del total de 576 curvas, se conoce actualmente la geometría de 72 de ellas, y se ha realizado el vídeo de 102.

El cuestionario se difundirá por medio de correo electrónico y redes sociales a todo tipo de personas, solicitando su redifusión con el fin de lograr el mayor impacto posible. Previamente a ello, se realizarán varias pruebas de menor difusión, con el fin de recibir retroalimentación acerca de la idoneidad de las preguntas y del método. Estas iteraciones permitirán pulir el cuestionario, así como analizar el comportamiento de la base de datos con una cantidad de datos cada vez mayor. 
Tras el cierre de la recogida de datos, se procederá a su filtrado, clasificación y análisis. Este último será muy dependiente del número de respuestas obtenidas, por lo que no se han planteado todavía metodologías de análisis, más allá de un primer análisis exploratorio.

\section{AGRADECIMIENTOS}

La presente investigación es parte del proyecto "CASEFU - Estudio experimental de la funcionalidad y seguridad de las carreteras convencionales" (referencia TRA2013-42578P), que pretende actualizar el modo en el que se diseñan las carreteras convencionales en España atendiendo a criterios de operación, seguridad y funcionalidad. Dicho proyecto está financiado por el Fondo Social Europeo y el Ministerio de Economía y Competitividad.

\section{REFERENCIAS}

AASHTO. (2010). Highway Capacity Manual. Transportation Research Board CAMACHO-TORREGROSA, F.J., PÉREZ-ZURIAGA, A.M., CAMPOY-UNGRÍA, J.M. GARCÍA, A. Y TARKO, A. (2015). Use of Heading Direction for Recreating the Horizontal Alignment of an Existing Road. Computer-Aided Civil and Infrastructure Engineering, vol. 30(4), pp. 282-299

FITZSIMMONS, E., SOULEYRETTE, R. Y NAMBISAN, S. (2013). Measuring Horizontal Curve Vehicle Trajectories and Speed Profiles: Pneumatic Road Tube and Video Methods. Journal of Transportation Engineering, vol. 139(3), pp. 255-265.

MINISTERIO DE FOMENTO. (2016). Instrucción de carreteras 3.1 IC "Trazado". Ministerio de Fomento de España.

OTTESEN, J. Y KRAMMES, R. (2000). Speed-Profile Model for a Design-Consistency Evaluation Procedure in the United States. Transportation Research Record, vol. 1701, pp. $76-85$.

PÉREZ-ZURIAGA, A.M. (2012). Caracterización y Modelización de la Velocidad de Operación en Carreteras Convencionales a partir de la Observación Naturalística de la Evolución de Vehículos Ligeros. Tesis Doctoral, presentada en la Universitat Politècnica de València. Accesible en: http://hdl.handle.net/10251/16701

SPACEK, P. (2005). Track Behavior in Curve Areas: Attempt at Typology. Journal of Transportation Engineering - ASCE, vol. 131(9), pp. 669-676 